Oberlandesgericht, sich in einer Pressekonferenz am 20. 12. 974 der Offentlichkeit zu stellen. Sie wiesen sämtliche Vorwürfe Dr. Kupkes an die Justız zurück und fanden seinen Tod *unerklärlich «. ${ }^{3}$

Ein Richter, der mit seinem Kollegen Dr. Kupke im "Gesprächskreis Frankfurter Richterø zusammengearbeitet hatte, kam in seiner persönlichen Erklärung im Rahmen der Trauerfeier zu einer anderen Einschätzung: Mit anderen gehe er in Kenntnis der Person Dr. Kupkes und der bekanntgewordenen Umstände des Todes dieses Mannes davon aus, daß der Richter nicht zuletzt an den Strukturen und Hierarchien der Justiz sowie an deren Methoden der Konfliktregelung zerbrochen sei.

Am 29. November 1974 hatte Dr. Kupke mit einer Exkursionsgruppe der Projektgruppe Mietrecht der Freien Universität Berlin, die ihn zur Mietrechtsprechung in Frankfurt befragen wollte, das nachstehende (mit einigen Kürzungen und Zusammenfassungen) abgedruckte Gespräch geführt. Es zeigt exemplarisch die Schwierigkeiten, denen gegenwärtig die Arbeit von progressiven Richtern innerhalb der Justiz ausgesetzt ist.

Walter Roth / Roland Vogt

\title{
Tonbandaufzeichnung eines Gespräches mit dem ver- storbenen Frankfurter Mietrichter Dietmar Kupke
}

"Ich möchte anfangen, mich selbst vorzustellen. Ich bin schon lange im Mietrecht tätig. 1970 bin ich erstmals aushilfsweise hier als Mietrichter hingesteckt worden bzw. abgeordnet worden, wie es so bei der Justiz heißt. Dann allerdings bin ich hier in diese ganze Mietrechtsentwicklung reingekommen, mit diesem Problem konfrontiert worden und habe auch an spektakulären Prozessen teilgenommen, so z. B. auch an dem Kettenhofwegprozeß. Der Kettenhofwegprozeß ist bekannt, ich habe damals zunächst mit Unterstützung der Stadt und des Wohnungsamtes einen Vergleich zustande gebracht. Ein Jahr später ist es dann zu einer schweren Schlacht gekommen, was ich eben verhindern wollte. Ebenso habe ich mich dann auch an der Universität mit Mietrecht befaßt, insofern als man aus studentischer Sicht an mich herangetreten ist, ob ich bereit wäre, im Rahmen einer losen Veranstaltung in der Universität über meine Erfahrungen zu berichten. Die studentischen Körperschaften der Universität haben es dann dem Dekanar gegenüber erreicht, daß meine Tätigkeit über cinen Lehrauftrag abgesichert worden ist. So halte ich seit 1972 ein Mietrechtskolloquium ab, in dem ich praktische Fälle, besonders auf die Frankfurter Verhältnisse und gerade auf momentane Aktualität bezogen, behandle oder neue gesetzliche Vorhaben, so z. B. das Sozialbindungspapier bespreche. Dazu haben wir Referenten geholt und auch andere Interessenvertreter zugezogen. Wir sind dann über das Mietrecht hinausgegangen und haben gesehen, daß Mietrecht, Baurecht und Bodenrecht unzertrennbar miteinander verbunden sind. Wir versuchen deshalb, auch weiter dahingehend tätig zu sein. Ich war inzwischen auch mal zwei Jahre aus der praktischen Arbeit weg. Ich bin erst seit zwei Monaten wieder als

3 FAZ vom 23. 12. 1974, - Die Oberen können sıch den Selbstmord nicht erklären. - Zum Tode ron Kupke s. a. die Berschte in der Frankfurter Rundschau vom 13., 14., 19., 20. und 21. 12.1974 . 
Mietrichter tätig. In der Zwischenzeit war ich auch am Schwurgericht in einem Prozeß tätig, wo es einen Knall gegeben hat, von dem Sie wahrscheinlich auch in Berlin gehört haben. Der Prozeß ist geplatzt, weil sich die Vorsitzende als befangen abgelehnt hat... Ich habe eine Presseerklärung abgegeben, indem ich auf emen Mißstand (Zwischenruf: Fasold-Prozeß), indem ich auf Grund des Pätsch-Urteils auf einen gravierenden Mißstand in der Frankfurter Justiz hingewiesen habe, nämlich die Überbelastung der Vorsitzenden Richterin, die noch durch zu große Kammergerichtstätigkeit uberfordert ist, so daß sie im Schwurgerıchtsprozeß, wo es immerhin um Kapitalverbrechen geht, wo hohe Straten auf dem Spiel stehen, sogar lebenslänglich, überfordert war. So hat sich die Vorsitzende Richterin nicht der Sache widmen können, was auch nach dem geltenden Recht und der Rechtsprechung unzulässig ist. Als alle Versuche, die Sache sonst zu bereinigen, nichts halfen, habe ich eine Presseerklärung abgegeben. Seitdem versucht man, mit gewissen repressiven Maßnahmen gegen mich vorzugehen, ${ }^{1}$ wogegen ich mich wieder wehre. Die Sache ist noch am Laufen. Ich fand die Presseerklärung durch das Pätsch-Urteil und auch durch meine richterliche Unabhängigkeit voll gerechtfertigt, um eben diesen Prozeß zu retten. Das war problematisch, aber das wollte ich nur am Rande erwähnen.“

\section{Frage: Nach der richterlichen Unabhängtgkeit in Zivilprozessen}

* Zu dieser Frage möchte ich folgendes sagen: es ist ein unhaltbarer Zustand, daß ein Richter auf Probe von seinem Vorgesetzien jedes halbe Jahr beurteilt wird, ständig versetzt werden kann und von einem auf den anderen Tag entlassen werden kann. Und das alles ist auch in der Praxis vorgekommen. So ist ein Ruchter gefeuert worden, aus Gründen, die meiner Meinung nach ganz wo anders lagen, wogegen zwar jetzt prozessiert wird, aber wahrscheinlich ohne Erfolg. Und gerade in diesen Dingen ist es ja so, daß ein Richter auf Probe stark unter dem Druck der Verwaltung steht, da er ja benotet und beurteilt wird. Es gibt auch Anwälte, die versuchen, daraus Kapital zu schlagen, zu ihren Gunsten Druck auszuüben, ja auch manchmal, wenn ein Urteil ihnen nicht paßst, einfach Dienstaufsichtsbeschwerde erheben. Sie schreiben dann irgendwie rein: »Ja, er ist unfähig oder hat eine linke Gesinnung “ oder ähnliche Dinge. Bei Richtern auf Probe wird so etwas teilweise auf unglaublich skrupellose Weise getan. Die gehen davon aus: Der wird sich in entsprechender Weise sowieso nicht wehren, da er dann mit der Verwaltung Schwierigkeiten kriegt; denn die Verwaltung will Ruhe haben, will nicht, daß der Richter da in entsprechender Weise vorgeht. Dasselbe würde die Verwaltung bei fest angestellten Richtern nicht wagen. Es ist also ein Unding, daß von den fünf Mietrichtern, die wir in Frankfurt haben, drei Richter auf Probe sind. "

\footnotetext{
1 Aufgrund der Presseerklärung von Kupke rtchtete der Präsident des Amesgenchts Frankfurt/M. am 22. August $197+$ an alle Richter und Rechtsptleger seines Amtsbereiches ein Schreiben, in dem es u. a. hief: [...] - Der Beante muß sein Recht auf freie Meinungsäußerung hinter die durch sein Trueverhältnıs zum Stast beyründeten besonderen Pllichten zurücktreten lassen, (vgl. näheres Komm. Bonner Grundgesetz von Mangoldt-Klenn, Ausgabe 1957, Bem. IX te). Es widerstrerte dem wohlbegründeren lnteresse des Staates, wenn dienstliche persönliche Differenzen unter Richtern. Fehler oder. Wängel in der Arbeitsweise der Jusıtzbehörde sm allgemeinen oder in bestımmten Abteilungen durch Pressenouzen bekanntgemache werden. Von diesem Grundsatz mag es die Ausnahme geben, daß die persönliche Integritä des Verfassers zur Wahrnehmung seines eigenen Interesses durch enne Pressemutteilung gerechtfertigt werden soll. [...] Ganz abyesehen davon, dais derartige Pressenotizen vermeldbare Unruhe in die Bevölkerung bringen, können sıe aber auch Unzufriedenhert in die Reihen der betroffenen Bediensteten, die nicht einmal vorher gehört worden sind, tragen, was wiederum zu etner Störung des allgemeinen Betrebsklimas führen hann.- [...]
} 
Frage: Wie das denn aussehe, wenn es sich um Richter auf Lebenszett handle. Frage wurde zugespitzt: $o b$ aus der mieterfreundlichen Rechisprechung irgendwelche Nachteile, irgendwelche Repressionen von seiten der Justizverwaltung $z u$ befürchten seien.

-Ich bin im Moment etwas befangen, weil ich Repressionen aus einem anderen Grunde ausgesetzt bin. Eben aus dem Grunde, weil ich mich mit dem Problem auseinandergesetzt habe, das die Meinungsfreiheit des Richters und Treuepflicht gegenüber dem Staat betrifft, weil ich eben diese Presseerklärung abgegeben habe im Sommer, die dem Justizministerium nicht gepaßt hat, d. h. sie haben eine Vorermittlung gegen mich eingeleitet, der ich mit Gelassenheit entgegensehe. Es ist zunächst einmal überhaupt unerwünscht, wenn Beamte und Richter an die Offentlichkeit treten. Da reagiert der Staat unheimlich gereizt, wenn man irgendwelche Mißstände kritisiert, selbst wenn man das in einer sachlichen Form tut, selbst wenn man sich dazu genötigt fühlt. Das ist der eine Punkt.

Jetzt, was die Mietrechtsprechung angeht, hütet man sich natürlich, äußerlich den Anschein zu erwecken, man greife in die Rechtsprechung ein. Außerlich hütet man sich, aber es existiert eine Repression. Es besteht die Gefahr, will ich mal sagen, daß so eine indirekte Repression ausgeübt wird, die schlecht nachzuweisen ist und die schlecht zu belegen ist, die man aber doch zu spüren bekommt, wenn man auf Dauer eine Rechtsprechung oder eine Art der Verhandlungsführung an den Tag legt, die nicht gewünscht wird. Ich darf z. B. erinnern, damals im Jahre 1972 habe ich über die Räumung des Hauses Kettenhofweg verhandelt. Ich habe da mit den Hausbesetzern gleich einen Vergleich zustande gebracht. Es war ein voller Sitzungssaal, und ich muß sagen, im allgemeinen hat sich das Publikum diszipliniert verhalten, aber mir wurde vorgehalten, ich hätte einiges durchgehen lassen, daß geraucht wurde und daß CocaCola-Flaschen dagelegen hätten usw. Eigentlich hat man mir vorgeworfen, daß ich überhaupt mit Hausbesetzern verhandle. Das hat man mir natürlich nicht direkt gesagt, aber ich habe das zu spüren bekommen. Ich bin auf noch größeren Unwillen gestoßen, als ich den Schwurgerichtssaal nahm und fünfhundert Zuschauer da rein ließ. Das ist auf noch größeren Unwillen gestoßen und man bekommt das dann eben so zu spüren. Natürlich werden die sich hüten, offen zu sagen: "Beförderungssperre", sondern es werden dann andere Gründe vorgeschoben. Es gibt da nicht nur Beförderungssperren, sondern es gibt dann auch Dinge, mit denen man einen ernannten Richter schikanieren kann, selbst wenn er nicht befördert werden will. Man läßt dann eben plötzlich sein Dezernat anwachsen, oder wenn man in Urlaub ist, kriegt man die mieseste Urlaubsvertretung. Es passieren dann so ganz komische Geschichten, es werden Anträge abgelehnt, die bei anderen nicht abgelehnt wurden. So ist das schon, wenn man eine gewisse Toleranzschwelle, die also von der Verwaltung gewünscht wird, überschreitet. Dann bekommt man den Unwillen der Verwaltung indirekt zu spüren, und die Verwaltung, die Justizverwaltung hat so eine Form entwickelt, die also teilweise besonders beispielhaft ist, insofern als sie in gewisser Weise hinterhältig ist. Damit man sie nicht zu fassen bekommt, werden also Dinge so gemacht, daß man sie nicht richtig nachweisen kann, z. B. auch bei Beschwerden.

Es ist so, daß die Mietrichter dermaßen überlastet sind auch mit Kleinkram, so daß sie quasi zum Teil wie Fließbandarbeiter arbeiten müssen und sich dadurch den sozialen Problemen nicht richtig widmen können, da sie zum Beispiel nicht 
grenügend Zeit haben, sich bei Ortsterminen die Häuser anzusehen oder ähnliche Dinge durchzuführen. Wenn man sich aus solchen Gründen bei der Verwaltung beschwert, dann machen die erstmal gar nichts. Da sagen sie, Sie haben nicht soundsoviel Nummern (d. h. Urteile, Anm. d. Red.). Die zählen ja nur die Urteile, was da drin steht, interessiert sie gar nicht. Daß die Richter vergleichen, das ist ja viel wichtiger, weil sie dadurch irgendeinen Frieden stiften. Das interessiert auch überhaupt nicht, die interessiert nur die Zahl der Urteile, ob sie da 3 oder 20 Sätze oder gar nichts reinschreiben, nur daß es besteht, das wird gezählt. Und daran wird der Richter gemessen, nach dem Output-System. Der Richter, der die meisten Urteile produziert, gilt also neben der Examensnote als meist beförderungswürdig. Man spricht von der Belastbarkeit - immer wieder kommt das Wort Belastbarkeit - das ist ein Schlüsselproblem. Der Richter muß belastbar sein, immer wird die Belastbarkeit des Richters geprüft. Und am liebsten wird gesehen - früher rühmte man sich ja damit -, wenn von einem Tag zum anderen ro Urteile ausgestoßen werden. Das gilt als besondere Glanzleistung. Dieser Kritikpunkt steht auch im Zusammenhang mit meiner Presseerklärung im Schwurgerichtsprozeß. Da war die Vorsitzende noch an 3 anderen Spruchkörpern tätig und hatte dazu am laufenden Band noch Strafurteile ausgestoßen. All diese Dinge habe ich bemängelt und bin damit in ein Wespennest innerhalb der Justiz gestoßen. Die Justizverwaltung wünscht also belastbare, leistungseffiziente Richter, die ein großes Ausmaß an Ausstoß haben und legt keinen Wert auf die Qualität der Rechtsprechung, ob diese menschlich ist und ob sie sich mit den menschlichen und sozialen Problemen richtig befaßt. Deshalb gewährt sie den Richtern nicht ausreichend Zeit. Wenn man sich dagegen wehrt, wenn man sich innerhalb der Verwaltung wehrt, geschieht nichts. Macht man dann den nötigen Druck oder geht man dann an die Offentlichkeit, dann wird man diesen indirekten Repressionen ausgesetzt und es passieren Dinge, die sonst nicht passieren; denn man macht ja immer mal so kleine Fehler, z. B. im technischen Ablauf, daß man mal irgendeine Verfügung andersherum macht als sonst, was normalerweise nie beanstandet wird, wird plötzlich als Grund für eine Aktenprüfung genommen unverhofft. Sie stellen irgendeinen kleinen Fehler fest und es besteht dann die Gefahr, daß das in die Personalakten kommt. Dies sind dann die kleinen Schikanen, denen sie ausgesetzt sind. Das kommt dann immer in regelmäßigen Zeitabständen, da werden dann Aktenprüfungen vorgenommen und es wird dann festgestellt, ob sie einen Fehler gemacht haben, während normalerweise jahrelang überhaupt nicht geprüft wird, was sie mit ihren Akten machen, ob sie sie verschlampen oder sonst etwas damit machen. Aber wenn sie da mal unangenehm aufgefallen sind, dann müssen sie damit rechnen, daß sie dauernd beobachtet werden. Sie werden nucht danach beobachtet, ob sie sich um die sozialen Probleme kümmern, sondern es wird darauf geachter, ob sie die Akten richtig bearbeiten, ob sie die Verfügungen sachgerecht bearbeiten, wie das nach dem Preußischen Recht aus dem vorigen Jahrhundert überliefert ist. Ob sie das richtig machen und wieviel Urteile sie produzieren, darauf wird geachtet. Es wird Statistik geführt, ganz peinlich: ach, der hat die wenigsten Urteile! Der ist nicht belastbar. «

Frage: Inwiewett versucht die Justizverwaltung, materiell auf die Rechtsprechung Einfluß zu nehmen?

(Diskussionsresultat: So plump arbeite die Justizverwaltung denn doch nicht.) 
Frage: Inwieweit über den Einfluß auf Versetzung und Beförderung eines Richters dann doch Einfluß genommen werden könnte auf den Inhalt der Recht. sprechung. Etwa: Dieser oder jener Richter produztert berufungs- und revistonssichere Urteile, die Urteile des anderen werden ständig aufgehoben.

"Man gibt den Amtsrichtern eine gervisse Freiheit, der Grund ist aber, daß man von den Urteilen des Amtsrichters nicht allzuviel zu befürchten hat, denn wenn das Urteil mit der Obergerichtsrechtsprechung kollidiert, wird es von der höheren Instanz wieder aufgehoben. Ich habe aber sehr interessante Erfahrungen gemacht, einerseits im Vergleich zwischen Amtsrichtern und andererseits mit meiner Tätigkeit als Richter am Schwurgerıcht. Man legt großen Wert darauf, daß die Schwurgerichtsurteile rechtskräftig werden und diese Schwurgerichtsrichter neigen dazu - es gibt ja nur noch die Revision in diesen Schrvurgerichtssachen, da kommen die Konflikte hinein -, die Dinge so schnell als möglich über die Bühne zu ziehen, um rechtskräftige Urteile zu erzielen. Es gibt Richter, die laufen herum und brüsten sich damit, daß alle ihre Urteile rechtskräftig geworden sind. Dieselben Richter verurteilen natürlich Kritik, die in die Richtung geht, die ich beanstandet habe, Kritik an dem riesigen Ausstoß von rechtskräftigen Urteilen, die zwar yom Bundesgerichtshof bestätigt wurden, meines Erachtens aber ihre Rechtskraft offenbar mit etwas zweifelhaften Methoden erhalten haben. Das ganze ist manchmal sehr zweifelhaft, da sie im Landgericht jedes Urteil revisionssicher machen können. Beim Amtsgericht schaut man deshalb gar nicht so darauf. Amtsrichter werden in dieser Beziehung nicht für so wichtig erachtet, weil die Masse sehr groß ist. Aber bei den Schwurgerichtsvorsitzenden, die wollen ja nachher Bundesrichter werden und Senatspräsident beim Bundesgerichtshof, die achten peinlich darauf, daß sie einen riesigen Output yon rechtskräftigen Urteilen vorweisen können. Das ist eine höchst bedenkliche Sache und hängt mit unserem System zusammen, daß es bei einem Hühnerdieb eine Berufung und eine Revision gibt, wenn aber einer einen Mord begeht oder wegen eines Mordes angeklagt wird, dann hat er überhaupt nur die Revisionsmöglichkeit. Aber ein Urteil revisionssicher zu machen, ist keine Kunst. $\mathrm{Da}$ wird bei denen peinlich darauf geachtet, ob ihre Schwurgerichtsurteile (und Kammergerichtsurteile) häufig aufgehoben werden oder nicht. Derjenige, der die meisten Urteile durchbringt, der hat dann die größte Chance, einmal Senatspräsident beim Bundesgerichtshof zu werden. *

\section{Frage: Nach der Interdisziplinarität}

(Angesichts des Konfliktfelds Mietrecht wurde Herrn Kupke die Frage gestellt, ob man denn diesen Konflikten voll gerecht werden könnte, wenn man rein mit juristischer Dogmatik an die Fälle herangehe. Wir hatten uns ja vorgestellt als eine Gruppe, die interdisziplinär arbeiten möchte. Herr Kupke ging auf diese Frage ein, indem er vor allem klarlegte, daß das bisher nicht möglich war. Früher in der Diskussion wurde übrigens gesagt, daß durch die Belastung des Richters dieser praktisch keine Zeit hätte, sich mit außerjuristischem Material, das an sich zur Lösung der Fälle nötig wäre, zu befassen.)

"Der Justizaufbauapparat ist darauf angelegt, nur gesetzeskonform nach Palandt etc. zu urteilen. Eine Erarbeitung und Ausweisung interdisziplinärer Art hat in der Rechtsprechung, von einigen Ansätzen abgesehen, überhaupt noch nicht Eingang gefunden. Daher arbeiten die Richter noch immer nach rein fachbezogenen Aspekten mit ihrem juristischen Handwerkszeug, wobei begün- 
stigend wirkt, daß das Beförderungssystem für Richter so aussicht, daß diejenigen befördert werden, die den meisten Output haben, und den meisten Output haben die, die nach Palandt und den rechtlichen Bestimmungen der Rechtsprechung urteilen, die sie eben griffbereit haben. Da gibt es also solche maßgeblichen Kommentare wie Palandt, Schmidt-Futterer etc. Hat eine Entscheidung einmal Eingang gefunden, dann wird nach dieser Einbahnstraße schnell judiziert, diese Art wird gewünscht. Diese Urteile haiten sich dann, weil auch die Obergerichte an dieser Rechtssprechung festhalten. So wird von vornherein jeder Versuch, eine Sache auszuweiten und über den eigentlichen normativen und rein formellen Bereich hinauszukommen, äußerst erschwert. Es kommt noch dazu, daß Grundlagen fehlen, die in die Rechtsprechung Eingang gefunden haben. *

(Wir kamen noch einmal auf die gerade für Studienanfänger, die sich am künftigen Berufsbild des Juristen, des Richters orientieren wollen, interessante Frage der Disziplinuerbarkeit von Richtern zurück. In diesem Zusammenhang kam noch einmal das Problem des Richters auf Probe auf den Tisch. Darüberhinaus wurde die Frage erörtert, die gerde aus Berliner Perspektive sehr wichtig ist, inwieweit man im Zusammenhang mit Stadtteilarbeit, z. B. schon als Student, Mieterberatung betreiben kann und vielleicht auch den einen oder anderen rechtsunkundigen Mieter vor dem Amtsgericht vertreten kann. Daran knüpfte sich die Diskussion um das sog. Rechtsberatungsmißbrauchsgesetz an.)

* Das Rechtsberatungsmißbrauchsgesetz ist in der Hitlerzeit entstanden, darauf muß man erst mal hinweisen. Und dann zweitens: Die Geschäftsmäßigkeit wird also darin gesehen, daß man dafür Geld nimmt. Leute, die das aus Idealismus tun, werden da völlig ausgeschlossen. Man betrachtet auch das alles als geschättsmäßig, weil sie dies laufend tun. Das ist geradezu eine Perversion der Geschäftsmäßigkeıt. Natürlich, die Anwälte sind sehr scharf daraut, daß das Gesetz eingehalten wird, weil ihnen sonst ihre Pfründe verloren gehen könnten, wenn da andere Leute als Konkurrenten auftreten. Dann muß man auch sehen, daß das Mietrecht doch von der Creme der Anwaltschaft, von den großen bekannten Anwälten vernachlässigt wird. Denn das bringt ja nichts ein. Die Anwälte arbeiten natürlich nicht geschäftsmäßig, sondern das ist eine wissenschaftliche Tätigkeit, die die Anwälte machen. Ja, ja eine Erklärung des Bundesfinanzgerıchts liegt hier vor. Aber wenn einer aus Idealismus Leute vertritt, dann macht er das natürlich geschäftsmäßig! Und dann droht die Gefahr, daß er erstmal ausgeschlossen und dann bestraft wird wegen Rechtsberatungsmißbrauch. Also, wer hier was mißbraucht, das ist wohl anders. Das hat eine Dialektik in sich selbst und bei verfassungskonformer Auslegung, das ist meine Auffassung dazu, halte ich das so, wer da eben nicht zu Erwerbszwecken tätig wird, fällt meiner Ansicht nach nicht darunter. Wenn man ein gewisses Repressionsschema erträgt, kann man als ernannter Richter stur sein und dagegen anlaufen. Allerdings wird jemand, der Rechtsberatung gibt, doch irgendwann zu Fall gebracht, weil dann die Verwaltung mit der Staatsanwaltschaft gegen ihn vorgeht und ihn dann rausschießt. Das ist dann der letzte Weg. Nochmal grundsätzlich zu der Frage des Engagements. Ich habe schon gesagt, als die Frage gestellt wurde, daß, wenn man etwas länger tätig ist, daß man dann sehr leicht resigniert. Es ist so, Sie können Engagement in einzelnen Fällen als Richter entfalten, können einzelnen Menschen helfen und auch sozial Schwachen zu ihrem Recht verhelfen, im Einzelfall einzelnen Personen, was auf der anderen Seite oftmals dazu führen kann, daß sie diese privilegieren gegenüber anderen, die zu einem anderen Richter kommen und das Pech haben, dann 
eben schlechter dazustehen. Insofern künnen Sie ein Engagement enttalten, haben dann eine gewisse Befriedigung und können sich sagen, hier dieser alten Frau habe ich gehoifen. İch habe also vernindert, daß sie aus dem Stadtteil vertrieben wird. Ich hatte da mal so einen Fall, der ist ja auch ewig durch die Presse gegangen. Es war ein rechtskräftiges Urteil ergangen, es sollte geräumt werden und ich ging hin mit einem Beschluß, da ist die alte Frau wieder in ihre Wolinung reingetragen worden. Da habe ich mich also stur gestellt und ich meine, in dem konkreten Fall hat das dazu geführt, daß die Frau dann von der Stadt eine Neubauwohnung gekriegt hat im Westend. Nachdem die Berichterstattung in der Presse nicht abbrach, hat Frau Jahnke sogar noch einen Erholungsurlaub von der Stadt bekommen für die Aufregung. Da hat man nun das Gefühl, gut, dieser einzelnen Frau hat man geholfen. Das ist ein gewisses Engagement im Einzelfall. Es ist natürlich die Frage - und da muß man resignieren -, ob sie als Richter eben insofern verändernd wirken können, daß sie das Recht, die Gesellschaft menschlicher machen. Da sind ihre Wirkungsmöglichkeiten, insbesondere auch als Amtsrichter, besonders gering, allenfalls werden da den Verfassungsrichtern Möglichkeiten gegeben, aber da kommen ja eben Leute nicht hin, die nicht so justizkonform sind. Es fällt manchmal in höheren Kreisen der Ausspruch, ein Amtsrichter ist im allgemeinen nur der, der eben faul oder reich ist und sich das leisten kann. Das sind so gewisse Sprüche in oberen Gerichten. Aber ich meine, einen Vorteil hat der Beruf des Amtsrichters, sie können alleine entscheiden. Sie können da ein gewisses soziales Engagement im Einzelfall entfalten. Wenn sie in der Kammer drin sind, in einem Kollegialgericht und das habe ich am eigenen Leibe erfahren, und die anderen Kollegen denken anders, da können sie reden, was sie wollen, da können sie gegen die Wand reden, sie werden stur überstimmt. Und wenn sie mit denen nicht auf einer Wellenlänge sprechen, ist ihr ganzes soziales Engagement umsonst. Bei der im Moment herrschenden Großwetterlage, hauptsächlich durch die BaaderMeinhof-Hysterie verursacht, wird dieses soziale Engagement immer schwieriger. Dazu kommt die Ermordung Drenkmanns, die natürlich unter der Richterschaft zu einer unheimlich starken Hysterisierung geführe hat.

Es ist besonders in Frankfurt leider eine Gegenentwicklung eingetreten. Wir hatten hier den Herrn Wassermann, der ein recht liberaler Präsident war und auch als Verfasser liberaler Schriften hervorgetreten ist. Er wurde durch Intrigen von hier vertrieben. Wir haben seither einen Präsidenten, der genau das Gegenteil ist, der geradezu ein Anti-Wassermann ist, der eine unheimliche Macht ausübt. Er ist im Prüfungsamt und läßt also erst einmal die Leute zittern, bis sie in den Justizdienst treten. Er übt eine enorme Macht aus, da er über die besten Beziehungen zum Justizministerium verfügt und hat hier eine absolute Gegenentwicklung eingeleitet, insbesondere beim Frankfurter Landgericht. Das hat natürlich eine starke Bedeutung, weil es in Frankfurt ja die oberste Instanz ist. Eine absolute Gegenentwicklung, eine konservative und eine autokratische, kann man sagen. Diese Entwicklung fängt schon bei den Assessoren an, die werden auf Vordermann gebracht und jedes Aufmupfen wird sofort mit Repressionen belegt. Das ist cine spezielle Frankfurter Komponente, die eine Reaktion auf das Wirken von Wassermann hier in den letzten Jahren ist und die zu starker Resignation Anlaß geben wird.*

Frage: Wenn Sie von Repressionen sprechen, dann sagen sie, -ste* üben Repression aus oder sie " haben erreicht, daß der Wassermann da versetzt worden ist. Wie seczt stch das durch und wer tst das "stea? 
Ja, das ist eben, wenn man so sagen will, das Teuflische. Es ist schwer durchschaubar, es wird auch schwer durchschaubar gemacht und das "sie " ist erstmal das Justizmanisterium und die Bürokratıe des Justizministeriums, die einen starken Einflußs hat.

Es ist so, wenn sie sich unliebsam gemacht haben, dann werden gleich 3 Leute auf sie angesetzt und sie sind nur einer! Diese Bürokratie des Justizministeriums, die in direktem Kontakt mit dem jeweiligen Präsidenten steht - da gibt es Oberlandesgerichtspräsidenten, Landesgerichtspräsidenten, die alle konform geschaltet sind zum justizministerium - übt natürlich sehr starken Einfluß aus. Sie, die Präsidenten, geben die Benotung für die Assessoren. Das geben zwar an sıch die Vursitzenden, aber der Präsident gibe dann die Gesamtbenotung, so daß ein Einfluls gewahrt bleibt. Bei Betörderungen geben sie die Benotungen, sie beruten das Präsidium ein, die Präsidien entscheiden zwar, aber die Präsidien machen in den mersten Fällen eben das, was die Präsidenten machen und diese handeln wiederum aufgrund der Anweisungen aus Wiesbaden, und das ist eine anonyme Bürokratie. Wer das nun im Einzelnen ist, das weiß man nicht so genau. Das ist eben eine gut ausgestattete, stark vorhandene kräftige Bürokratie. Herr Wassermann war eine Ausnahmeerscheinung, der paßte hier nicht in die Landschaft. Man hat ihm eben so lange zugesetzt, bis er sich eben dann nach Braunschweig abgesezzt hat. Man hat ihm durch Nadelstiche ständig Schwierigkeiten bereitet, das kann man machen, gerade als Landgerichtspräsident ist er ja auch der Weisungsgewalt unterworten, des Oberlandesgerichtspräsidenten und des Justizministerıms, da ist er ja nicht richterlich unabhängig, sobald er Präsident ist. Das Wichtıge ist, es gibt eine bestimmte Gruppe, man kann fast sagen, ein Machtkartell von Senatspräsidenten und beförderten Richtern, die da mitmachen und diese Sache unterstützen. Man muß dazu sagen, die «sie", das ist sehr wichtig und th habe $j$ a mit den "sie" zu tun und merke den großen Bruder, der da so im Hintergrund ist. Das ist eine Bürokratie, die nicht nach außen in Erscheınung tritt, das ist die Ministerialratsebene, Ministerialdirigentenebene usw., die recht gut besoldet ist, deren Namen sie aber nicht kennen, die im Anonymen bleiben, eine Fülle von Verfügungen und Vorarbeiten machen und alles dirigieren und dann, wenn das Ministerium einen Richter promovieren lassen will, also hervorheben, hochheben will, wenn es meint, der lietert genug Urteile ab - natürtich spielt erstmal die Examensnote eine Rolle und dann der Output, den er hat -, wenn er also genügend Urteile ausgestoßen und Belastbarkest hat, dann darf er promovieren. Das ist also geradezu lachhaft, wie dann immer gleich der Ausdruck Belastbarkeit auftaucht. Wenn er genügend belastbar ist, dann holt man den sich erst mal ins Ministerium als Hiltsarbeiter, der bleibr dann Richter. Da gibr es die schöne Bezeichnung Amtsrsclater oder Oberlandesgerichtsrat in Justizministerium, obwohl er reiner Verwaltungsbeameer ist. Wer dann dort seine Probe bestanden hat, der steigt entweder im Ministerium auf oder kommt zurück als Senatspräsident oder als Direktor usw. Das nennt man das dritte Staatsexamen. Oder man holt ihn an das Oberlandesgericht, an entsprechende Senate, wo er dann entsprechend weiter dressiert wird. Der beste Wegr aber geht über das Justizministerium. “

Frage: Ist das Justizmintsterium SPD oder FDP?

- W'elcher Minister im Moment dran ist, spielt keine Rolle, weil die Bürokratie - das muß man wissen - den Ton angibt. Der Minister ist auf die Bürokratie anyewiesen. Wir haben hier selbst liberale Minister gehabt und weniger liberale 
Minister. Wer das System geleitet hat, war völlig gleich, weil die Leute, die darüber bestimmen, anonym bleiben. Das sind die Ministerialdirigenten usw., die bleiben jeweils und die sind auch in den einzelnen Ländern gleıch, egal ob die CDU oder die SPD an der Macht ist. Die gehen in dem Land, in dem die CDU an der Macht ist, notfalls in die CDU und wenn die SPD an der Macht ist, dann gehen sie in die SPD, um sich entsprechend abzusichern. Die haben sich ja durch das System entsprechend hochgearbeitet. Sie haben als Hiwis im Ministerium angefangen, haben sich hochgearbeitet, die würden in Bayern in die CSU gehen, in Hessen in die SPD, in Berlin auch. Das sind diese Leute, diese Bürokraten und Technokraten, die im Hinterhalt, also im Dunkeln dirigieren und alles ordnen und verwalten und wenn Sie sich mit dieser Macht anlegen, also da kann ich Sie nur warnen."

Frage: Das härt stch unheimlich restgnierend an, wenn man die ganze Represston von den Leuten hört und Sie haben gerade die Referendararbeitsgemennschaft erwähnt. Wie ist es da mit anderen Formen, sich zu wehren, einmal gegen diese komische dunkle Macht da irgendwo, die keiner richtıg fassen kann. Ist es tatsächlich so, daß man die nicht irgendwie in Griff kriegen kann, oder ist da auch die Angst drun, die nambaft zu machen und eine andere Frage, gibt es Ansätze unter Richtern, daß man sich vielleicht kollektiv wehrt oder tst das immer eine individuelle Entscherdung?

"Ich möchte dazu sagen, da ist natürlich ein Rückschritt eingetreten. Das Jahr $68 / 69$, da war dies alles noch wesentlich liberaler. Das war eine Zeit in Hessen, das haben Sie vielleicht von Berlin nicht so verfolgt, da waren einige fortschrittliche Leute - der Richterbund ist natürlich auch eine Organisation, da sind viele Konservative drin -, aber da waren einige Leute da, die haben gegen diese anonyme Bürokratie angestürmt und haben einfach erst einmal die Frechhert besessen - was damals undenkbar war -, gegen diese Leute zu klagen. Einfach den eigenen Arbeitgeber zu verklagen und sogar dann zu vollstrecken, das war etrvas vollkommen Neues. $D_{a}$ haben sie sogar einmal bei einem Präsidenten Taschenpfändung durchgeführt. $\mathrm{Da}$ gab's so eine Prozeßberwegung, einè gewisse Gruppe hatte sich zusammengetan gegen diesen anonymen Machtapparat, der sich also ailerlei - ich will ein bißchen vorsichtig sein - ziemlich viel angemaßt hat und den Leuten ganz hübsch zugesetzt hat. $\mathrm{Da}$ sind folgende Fälle vorgekommen: $D_{a}$ gabs einen Jugendrichter, der hatte sehr schwierige menschliche Fälle, auch Kinder, die ihren Müttern entzogen werden sollten usw. und der hat sich also darüber beschwert, daß bei dieser Vielfaltarbeit absolut nichts zu bewältigen wäre, und daß er es mit seinem Gewissen nicht mehr vereinbaren könnte, in dieser Weise zu arbeiten. Er ging in Urlaub und wie er wiederkam, war er weg rom Dezernat. ohne daß man ihn geiragt hätte, bums, weg war er. Der hat sich gewehrt mit enner Gruppe zusammen, die waren auch im Richterbund, dagegen und auch gegen andere Mißstände und die haben damals einen regelrechten Kampf hier mit der Bürokratie angefangen in den Jahren 66/68/69. Da war alsn ein gervisser Hoffnungsschimmer, da hatte sich eine Gruppe zusammengefunden und die hatte dann Unterstützung erhalten, als der Wassermann nach Frankfurt kam. Wir hatten auch mal eine kurze Periode mit einem relativ liberalen Justizminister namens Strelitz, den hat man inzwischen abgeschossen, so würde ich das nennen. Das war mal so ein gewisser Lichtschimmer. Ich will nur sagen, es gibt gewisse Perioden. wo etwas zu machen ist. Damals sind tatsächlich einige Dinge beseitigt worden. Die Büro- 
kratie mußte zurückstecken, sie mußte 2 . B. etwa sog. "schwarze Eintragungen a aus den Personalakten streichen etc. Das war damals möglich. Das ist jetzt nicht mehr möglich! Das war eben enne Anderung der Großwetterlage, die 68/69 hier in Hessen liberal war. Da haben andere schon mit der geballten Faust in der Tasche dagesessen, um hier enne Änderung herbeizuführen. Die Bürokracue blieb natürlich, die hat sich erst mal geduckt, sich dieser Entwicklung angepaßt. Als dann alle die weg waren, hat man zum Schlag, zum Gegenschlag ausgeholt und hat mit den Leuten abgerechnet. U.a. hängt damit auch der Rausschmı eines Assessors zusammen, der sich als Assessor stark engagiert hatte, und eben damals ziemlich sıcher war, weil Leute da waren, die ihn abgedeckt haben. Ich will mal sagen, da ja im Moment die ganzen Baader-Meinhof-Geschichten dazukommen, spielt eine Hysterisierung der Justiz eine noch vie! größere Rolle, als die der Bevölkerung. Das hat hier in Frankfurt schon mit den Demonstrantenprozessen angefangen, die waren hier sehr zahlreıch und haben zu dem Ergebnis geführt: Alle, die irgend etwas in Richtung Liberalisierung und Vermenschlichung getan haben, wurden als Anarchisten und Staatszerstörer denunziert. Wenn man irgend etwas kricisiert un der Justız, dann heißt es schon: man fällt der Justiz in den Rücken und hilft den Anarchisten und Ähnlichen. Eine unglaubliche Hysterisierung hat da eingesetzt. Nachdem man dann Wassermann und so vertrieben hatte, hat man bewußt Leute hingesetzt, von denen man wußte, die machen das richtıg. Die bringen die Richter zur Raison, die bringen die Referendare zur Raison. Man hat in dem Moment, als Wassermann wegging, sofort bei den Referendaren angesetzt. Wassermann hatte ja recht gehabt, und hatte vor allen Dingen auch seinerseits bei den Referendaren angesetzt, hat die Reterendararbeit intensiviert, hatte es schon in Berlin beim Kammergericht getan, hatte es hier in Hessen gelesstet, hatte auch die Mitwirkung der Referendare und die Artikulierung ihrer Forderungen, auch politischer Forderungen, weitgehend zugelassen. Es gab damals Richterversammlungen, Referendarversammlungen und all diese Dinge, die aber in Wiesbaden innerhalb der Bürokratie gerade zu schockierend gewirkt haben. Deswegen mußte Wassermann hier über die Klinge springen. Ich meine, jetzt in Niedersachsen mul3 er viel vorsichtiger agieren, sonst ereilt ihn da das Schicksal auch.

Das Erste, was hier war, als das neue Regime anfing, daß man die Freiheit der Referendare erst mal beschnitt, und alle, die dann aufmuckten, in Versammlungen Forderungen artikulierten und gegen bestimmte Mißstände auf die Barrikaden gingen, zu der Zeit als die Referendare noch Schönfelder für ihre Ausbilder einordnen mußten. Das wurde öfentlich angeprangert und alle, die diese Dinge anprangerten, die hat man dann gestrietzt, die hat man dann in Kammern gesetzt, wo sie die miesesten Ausbilder kriegten und dann noch schlechte Noten. Die mulbten wie die Wilden arbeiten. Die Note spielt ja eine große Rolle, weil man sie als Reterendar zu einem Drittel für's Examen angerechnet bekommt. Auf diese Weise hat man es verstanden, innerhalb relativ kurzer Zeit, die Referendare so zlemlich zu disziplinieren. Es gibt noch einige, die versuchen hier aufzumucken. Aber es ist ja su, als Referendar ist es noch nıcht ganz so schlimm, als wenn man Assessor 1st, weil man da sagen kann; gut, nachher kann der Anwalt werden und etwas mehr Rechte hat der Referendar noch im Falle eines Rausschmisses. Er gibt ja Entscheidungen, die besagen, der gesamte Ausbildungsweg gehört zur Ausbildung. Da hat der Staat das Ausbildungsmonopul, und der Referendar muß scine Ausbildung beenden können. Deswegen ist es schwieriger, emen Referendar als emen Assessor rauszuschmei- 
ßen. Aber es ist dann natürlich so, daß er nicht in die Justiz übernommen wırd. Er geht dann als Anrvalt. Desivegen hat der Referendar noch einen etwas größßeren Spielraum als der Assessor.

Die Ze1t als Assessor dauert vier Jahre. Den nächsten Schritt, den sie gemacht haben, war, einen Assessor nach vier Jahren mit Getöse rauszuwerfen. Wenn einer vier Jahre Assessor ist, dann schmeißt man ihn nicht raus, dann muß man ihn ernennen. Man hat den letzten Tag abgewartet und ihn dann rausgeschmissen. Der kämpft! Er ist jetzt beim Bundesrichterdienstgericht und hat mindestens schun 8000 DM an Prozeißkosten. Es ist natürlich eine Existenzfrage, die auf Sic zukommt. Das müssen Sie sich überlegen. Es ist eben so, nachdem die ganze Disziplinierung stattgefunden hat, was im Moment der Fall ist. Das geschieht immer wieder neu, wenn irgend etwas passiert. Manche freuen sich darüber, wenn irgendwo eine Bombe fliegt. Dann kann wieder eine Atmosphäre geschaffen wcrden, in der jedes Engagement, vor allem, wenn es in Gruppen geschieht, eben gleıch Repressionen auslöst. Aber es gibt die Möglichkeit zu hoffen, daß es mal wieder anders wird, daß sich die Emotionen legen. . Lernen, Widerstand zu leisten!

(Um noch einmal nachzufragen, ob die Fähigkeit Widerstand zu leisten nicht doch teilweise im justıziellen Bereich, auch im Bereich der Rechtsprechung gegeben ist. Da sind Begriffe aufgetaucht, die uns gerade in der Vorbereitung dieser Exkursion und jetzt wieder $1 \mathrm{~m}$ Gespräch aufgefallen sind. Das war einmal dieses "geschäftsmäßig" im Rechtsberatungsmißbrauchsgesetz, da meinen wir, fordert die Zivilcourage eines Richters tatsächlich einmal, das nach seinem Wortsinn zu benutzen, zumal es sich um eine Sanktion handelt, die zu Unrecht steht, denn da darf man doch nicht Recht gervissermaßen über den Wortgebrauch hinaus mißbräuchlich auslegen, daß man sagt regelmäßig und eben nicht darauf absteilt, ob der ein Honorar nimmt oder nicht. Das ist doch auslegungsmißbräuchlich! So gibt es auch Beispiele im Mietrecht (Benachteiligung von Ausländern), in Ausländerrecht und Demonstrationsrecht (friedlicher Sitzstreik wird als Gervalt und damit als Nötigung ausgelegt), wo das, was man an der Uni gelernt hat, in bestummten Situationen völlig über Bord geworfert wird. Die Fähigkeit, sich gegen solche Mißstände zu wehren muß gelernt werden.)

"Ich meine auch, wir sollten dic Fähigkeit lernen, Widerstand und Beharrlichkeit zu leisten und auch derartige Großwetterlagen, wie sie jetzt bestehen, durchzustehen und nicht zu kapitulieren. Man sollte sich davor hüten, sich in dieser Dezernatsarbeit zu verschleißen und den Kern dabei zu übersehen, daß eben durch dieses unmenschliche System, das die Justiz in vielen Bereichen, insbesondere in weiten Bereichen des Strafrechts und des Strafvollzugs, darstellt - aber auch in denen des Mietrechts - bei eben dieser Fließ̧bandarbeit die Richter gar nicht merken, daß sie Unrecht schaffen. Dafür muß man sich unbedingt die notwendige Zeit nehmen, mul3 man unter Wahrung der gewissen Vorsicht - insbesondere als Richter auf Probe, was betont werden muß - die Fähigkeit zum Widerstand und vor allem zum Durchhalten erlernen. Auf der anderen Seite steuert die Bürokratie diesem Anstoß entgegen, und zwar nach eincm Selektionsprinzip, da die unbedingt verhindern wollen, daß sich Gleichgesinnte zusammenfinden. Wenn die dann tatsächlich merken, da existieren einige Gleichgesinnte und das findet auch in ihrer Rechtssprechnung Niedersclilag, dann trennt man das Ganze. Es wird ja schon jetzt bei der Einstellung 
in den Justızdienst praktiziert. Die haben wieder genügend Bewerber. Sı schrauben jetzt erst mal die Noten hoch - 2,6 ist in Hessen die Einstellungsnote. Sie suchen sich die Leute ganz genau raus. Aufstieg als Disziplinierungsmittel. Ich muß doch wieder an die goldenen 68er, 6ger Jahre anknüpten: man hatte ja gehofft, daß aus dieser Referendarbewegung ene Richterbewegung und auch eın kontinuierlicher Nachschub für diese Richterbewegung werden würde. Diese Hoffnung hat eben getrogen, aus vielerlei Gründen, unter anderem aus dem Grund, daß man eine ganze Reihe von Berverbern mit fadenscheinigen Gründen abgewiesen hat. Man wollte sie nicht haben, weil sie eben nicht justizund systemkontorm sind!

Dann macht man noch tolgendes, das ist das zweit-diabolische, daßs man Leute, die noch autmucken, von denen man aber das Gefühl hat, die kann man umdrehen, daß man irgendwie versucht, diese Leute zu avancieren. Man sagt denen also: geh mal da an das Gericht, man sagt ihnen: sie sind doch ein guter Mann usw. Dann steckt man sie in eine Kammer und gibt ihnen, wie man so schön sagt, die Nestwärme. So werden sie in die Kammer integriert: man muß zusammen Mittagessen gehen und lauter solche Sachen, da gibt es, spöttisch sagen wir, den Storchengang, da geht der Vorsitzende vorne weg, dann der erste Bessitzer und hinten der Assessor. Da werden die Leute in die Kammer gesteckt, oft sucht man sıch dazu progressıve Leute aus, wo man das Gefühl hat, na ja, mit der Zeit, errecchen wir das schon im Guten. Man sagt ihm: sie sind duch gur usw. So dreht man die Leute emfach um! Da werden manchmal, das ergibt sich ohne weiteres, ohne daß man den Leuten einen Vorwurf machen kann, Leute nach einigen Jahren völlig verändert. Das sind plörzlich ganz andere geworden: sie finden überhaupt nichts mehr schlecht, finden alles gut und denunzieren die, die noch kritisieren, als St inkerer."

\section{Psychologische Kriegsführung und Existenzbedrobung ais Repressionsmitcel}

"Die da ,Obenc, die so was machen, die haben auch gelernt, wie sie diesen Bewegungen entgegensteuern. Vor allem wollen sie die, die Widerstand leisten, sıch gegen das System stellen, isolieren. Sie rerlektieren darüber: wenn einer isoliert ist, dann geht er nervlich kaputt. Wir werden den pieksen, den werden wir schikanieren, der muß isoliert sein, der darf ja keine Kontakte bekommen.

Diese gewisse Entwicklung in Hessen, die positive Entwicklung 68/69 war nur möglich, weil sich da eine Gruppe zusammengetan hat und die ist inzwischen auscinandergebrochen. Der isolierte Widerstand ist deshalb sehr gefährlich. Das sprechen die ganz offen aus da-oben, das haben die inzwischen wohl von der Psychologie gelernt, daß, wenn man Leute isoliert und sie dann jahrelang drangsaliert und im Ungewissen hält usw., daß man die Leute dann zur Frustration bringt. Und wenn man das Getühl hat, dałs sich irgendeine Gruppe zusammentut, die dann da irgendwie Arbeit leistet, daß man dann alle möglichen Mittel einsetzt, um diese Gruppe auseinander zu bringen, dalß man also den einen an's andere Gericht setzt, oder an's Landyericht, oder daß man Leute rumdreht, um diesen Tendenzen entgegenzuwirken. Deswegen ist das alles kolossal schwerıg. Es wird zur Zent bei der Einstellung peinlich darauf geachtet, wen man einstellt, dals man ja nicht die Falschen einstellt. Also die Radikalenfrage spielt schon gar keine Rolle nehr, man gucks schon ganzlich außerhalb dieser Frage daraut, ob es nicht etwa einer ist, der privat widerspenstig ist oder zum Widerstand neigt. Wenn da emer, das kann ich ruhig sagen, wenn da einer 
ein parmal in den Personalakten stehen hat, er neige zum Widerspruch und so, dann können sie damit rechnen, daß im Moment bei dieser Großwetterlage dieser dann nicht in den Justizdienst enngestellt wird, weil sie sagen, aha, hier haben wir einen, der macht Stunk nachher. $D a$ jetzt die Bewerber quasi Schlange stehen, können sie sich die Leute sehr stark aussuchen. Da machen die natürlich von dieser Sache Gebrauch und das ist eben ganz gefährlich. Hier zeigt sich eine Existenzgefährdung, die den Leuten droht. wenn ste abgewiesen werden. Sie können höchsters noch in der Anwaltschaft unterkommen usw. Das ist eine Existenzangst bei den Assessoren, die Familie haben oder ähnliches. Wenn die rausfliegen bei der Lage auf dem Arbeitsmarkt im juristischen Sektor. der sichtbar schlecht ist hier in Frankfurt, bei einer Uberbesetzung von Anwälten usw. ist das eine Existenzbedrohung. Diese Dinge spielen auch eine große Rolle, die Existenzangst, die wird auch bewußt eingeführt, um all diese Dinge zu ersticken.

Interview und Aufzeichnung: Projektgruppe Mietrecht, Staatliche Wohnungspolitile und Mieterselbsthilfeorganisationen an der FU Berlin.

\section{Die Praktiken des BND, oder: Was man aus dem Guillaume-Untersuchungsausschuß lernen kann.}

Eigentlich muß man G. Guillaume dankbar sein, hat doch sein Fall wenigstens ein wenig den Schleier der Geheimniskrämerei gelüftet, der traditionellerweise die Praktiken der bundesdeutschen Geheimdienste umgibt. Angeregt durch das öffentliche Interesse an dieser "größten Spionageaffäre in der Geschichte der Bundesrepublik ${ }^{1}$ förderte sogar der zu ihrer Aufklärung eingesetzte parlamentarische Untersuchungsausschuß Interessantes zutage. Dies ist umso erstaunlicher, als in Bonn jedermann weiß, daß parlamentarische Untersuchungsausschüsse äußerst untaugliche Aufklärungsmittel sind. Bundeskanzler Schmidt äußerte sogar, die Einsetzung eines Untersuchungsausschusses in dieser Affäre gefährde "das Ansehen des Parlaments schlechthin, einschließlich der CDU/CSU. Untersuchungsausschüsse hätten bisher noch nie dazu beigetragen, die Achtung vor dem Bundestag zu verstärken. ${ }^{2}$ Die Richtigkeit dieses Urteils stellte sich schnell heraus, denn sorwohl CDU/CSU als auch ihr nahestehende Zeitungen versuchten, die Gelegenheit vor allem zu dem Versuch zu nutzen, die SPD und sogar Verfassungsschutzpräsident Nollau als vaterlandslose Gesellen zu "enttarnen«:

- "Fraktionschef Herbert Wehner, so deutet die Opposition an, habe dem Kommunismus nie abgeschworen und SED-Chef Honecker von der bevorstehenden Enttarnung Guillaumes am 30. März 1973 unterrichtet.

- Günther Nollau, von Wehner geförderter Chef des Kölner Bundesamtes für Verfassungsschutz (BfV), so läßt die Reaktion in der Zeitschrift "Capital“" andeuten, sei ein Agent des Ostens.

- Marie, die tschechische Ehefrau des ehemaligen Kanzleramtsministers Horst Ehmke, so wird kolportiert, sei eine Ostagentin und

\footnotetext{
1 Der Spiegel (DS) $18 / 74$, S. 19.

2 Frankfurter Rundschau (FR) 12.6. 1974 .
} 